\title{
CONTROL OF SYSTEMS WITH ASYMMETRIC BOUNDS USING LINEAR PROGRAMMING: APPLICATION TO A HYDROGEN REFORMER
}

\author{
M. NAIB, F. TADEO, AND A. BENZAOUIA
}

Received 8 March 2005; Accepted 8 November 2005

This paper studies controller design for feedback systems in the presence of asymmetrically bounded signals, using a case study. An asymmetric objective functional is used to consider the asymmetrically bounded signals, which makes possible to derive a linear programming problem. Solving this LP makes possible to design controllers that minimize certain outputs, fulfilling at the same time hard constraints on certain signals. The method is presented by application to a hydrogen reformer, a system in petrochemical plants that produces hydrogen from hydrocarbons: a mixed sensitivity problem is stated and solved, with an additional constraint given by the asymmetric limitations on the magnitude and rate of the control signal, and the asymmetricity in the disturbances.

Copyright (C) 2006 M. Naib et al. This is an open access article distributed under the Creative Commons Attribution License, which permits unrestricted use, distribution, and reproduction in any medium, provided the original work is properly cited.

\section{Introduction}

In many control engineering problems, there are constrained variables (in magnitude, rate, acceleration, etc.), whose limits are not symmetric with respect to the nominal working point. This paper studies a novel extension of the $\ell_{1}$ control theory, to directly handle the asymmetrical aspect of bounded signals.

The topic of designing control systems that maintain stability (and performance) in the presence of these constraints is a topic of continuing interest (see, e.g., Eun et al. [7], Mesquine et al. [9], and references therein), because real plants always present constraints on signals (e.g., there is always saturation of the control signal due to the limited range of the actuators). Most of the approaches proposed in the literature are based on constraint avoidance: preventing the saturation, the closed-loop system stays in a region of linear behavior.

From the available approaches, we select the $\ell_{1}$ norm optimization concept (Dahleh and Diaz-Bobillo [3]), because the synthesis problem is solved using linear programming, and applications to real process have already been published (Malaterre and Kammash [8]; Tadeo and Grimble [14]; Tadeo et al. [16]), albeit for the symmetric case. In 
Vidyasagar [17] this problem was first formulated. Some basic results on this theory were presented in Dahleh and Pearson $[4,5]$. These results showed that the $\ell_{1}$-optimal control problem could be stated as linear programming problem. Since then, many other results and extensions have been presented (Blanchini and Sznaier [2], Sokolov [13]), but no extension to asymmetric signals has been published.

Then, this paper studies a novel extension of the $\ell_{1}$ control theory, to directly handle the asymmetrical aspect of bounded signals. The authors introduced this extension from a purely theoretical point of view in a previous paper (Naib et al. [11]), following the methods proposed for positive invariance (Dambrine et al. [6]; Benzaouia and Burgat [1]). This proposed extension to asymmetric signals is necessary because when the considered signal $u$ is constrained to evolve in an asymmetrical domain (i.e., signal $u$ is such that $-u_{\min } \leq u(k) \leq u_{\max }$, where $\left.u_{\min } \geq 0 ; u_{\max } \geq 0 ; u_{\min } \neq u_{\max }\right)$, in this case the peakto-peak norm of the signal $u$ (by definition, $\|u\|_{\infty}=\max _{k}(|u(k)|)=\max \left(u_{\max }, u_{\min }\right)$ ) gives a quite conservative information on the signal amplitude, so a new functional must be defined that takes separately into account the positive and negative deviations of the signals. This paper presents how the functional defined in (Naib et al. [11]) can be extended to a multiblock synthesis problem for a real engineering problem: the control of a hydrogen reformer plant in petrochemical industry, which prompted us the development of this technique.

The paper is organized as follows. Section 2 gives a description of the hydrogen reformer plant. Section 3 presents the reformulation of the $\ell_{1}$ optimal control theory taking into account the asymmetrical aspect of the considered signals. Section 4 is devoted to the application of this theoretical issue to the hydrogen reformer. Some conclusions end this paper.

\section{Description of the hydrogen reformer control problem}

The development of this extension of $\ell_{1}$ was prompted by a process control problem in an oil refinery, which had asymmetric constraints on the control signal and asymmetric disturbances. This process is the so-called hydrogen reformer: the objective of this system, in petrochemical plants, is to produce hydrogen from desulphurized hydrocarbons by catalysis (Tadeo and Grimble [14]). A schematic diagram of the system is shown in Figure 2.1. To generate the hydrogen, the hydrocarbons are mixed with superheated steam before entering the reformer tubes, where a nickel catalyst, heated at high temperature (about $750 \mathrm{C}$ ), produces the hydrogen. The high temperature that is reached to speed up the reaction is produced by burning fuel in the reformer. The fuel flow must be controlled to maintain the desired catalyst temperature, despite the presence of asymmetric disturbances and the fact that nominal flow is not near the middle of the allowed flow range.

The control system should maintain the desired catalyst temperature by modifying the amount of fuel that is fed to the reformer. The catalyst temperature and the fuel flow are measured for the implementation of the cascaded control structure shown in Figure 2.2.

In the real process, there are several disturbances, such as varying feed flow, fuel gas quality, steam temperature, and so forth. The most important disturbance is from the 


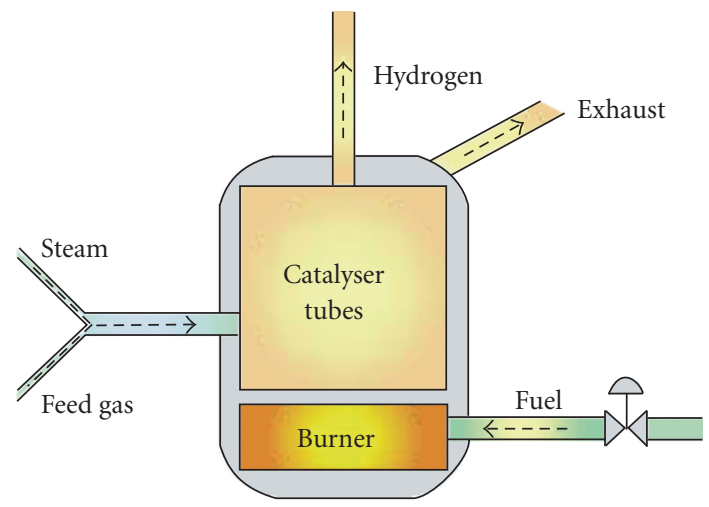

Figure 2.1. Diagram of hydrogen reformer.

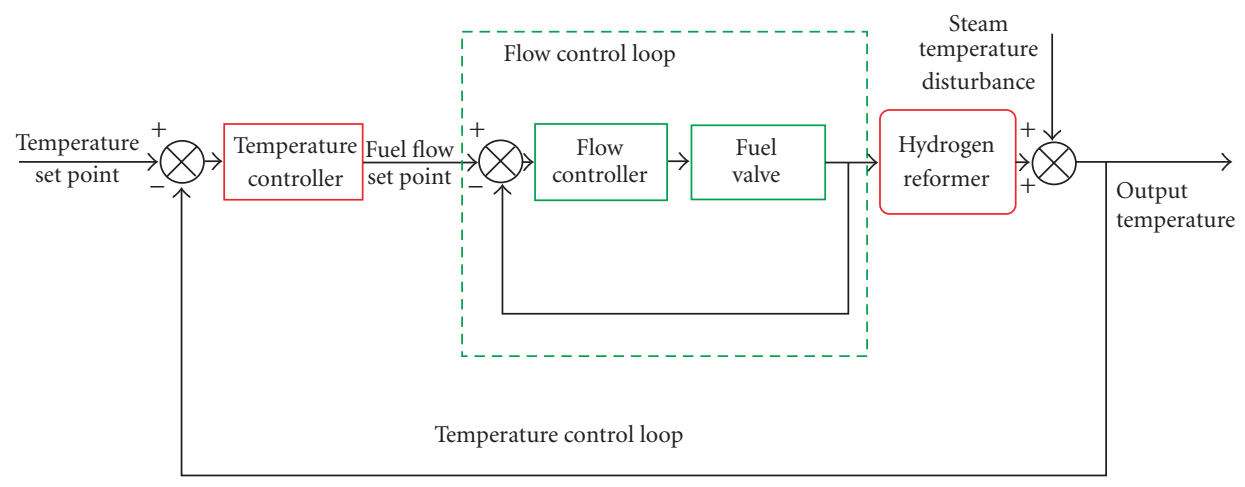

Figure 2.2. Control system in a hydrogen reformer.

steam temperature variation, which modifies the catalyst temperature directly. The control system must attenuate this (asymmetric) disturbance as much as possible by acting on the fuel flow set point (which is also asymmetric).

From a control engineering point of view, the control design problem is to design an advanced controller for this system that could improve the disturbance rejection properties, taking into account the nonsymmetric constraints on the amplitude and rate of the controlled variable (fuel flow).

First the existing control system was investigated and the slave flow control loop was considered adequate, so only the design of a master controller for the outer loop will be presented.

Summing up, reducing the existing slave loop, a schematic of the controller structure used for design is shown in Figure 2.3, which will be the topic of the rest of the paper. The main objective is to obtain tight control of the output, taking into account that the disturbance is asymmetric and the control signal is limited in amplitude nonsymmetrically. 
4 Control with asymmetric bounds of a hydrogen reformer

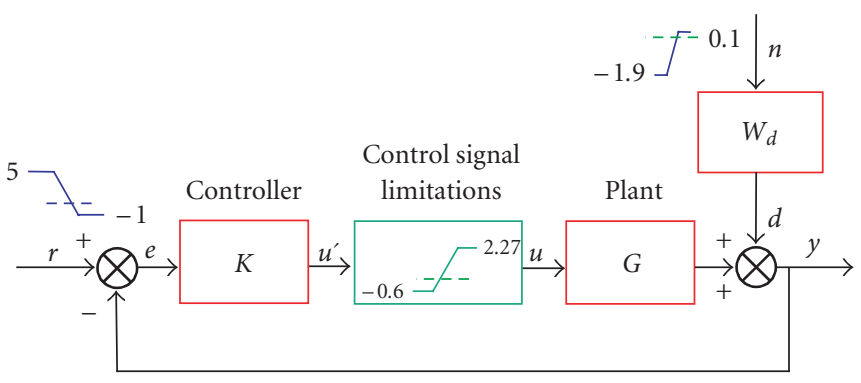

Figure 2.3. Control structure.

\section{3. $\ell_{1}$ analysis using asymmetric signals}

This section presents the objective functional proposed by the authors (Naib et al. [11]), which replaces the peak-to-peak-norm normally used in the $\ell_{1}$ analysis when asymmetric signals are used. For simplicity reasons, the method is presented for the singleinput/single-output case, although it is very simple to extend it to the multivariable case, as it is discussed later on.

3.1. Motivation. First, let us take a look in the $\ell_{1}$ norm of a transfer function $G$ representing a SISO discrete linear time-invariant system governed by an asymmetrical control signal $w(z=G w)$. It is well known that

$$
\|G\|_{1}=\max \frac{\|z\|_{\infty}}{\|w\|_{\infty}}, \quad \text { where }\|w\|_{\infty}=\max _{k} w(k),\|G\|_{1}=\sum_{i=0}^{\infty}\left|\phi_{i}\right|
$$

and $\left\{\phi_{i}, i=0, \ldots, \infty\right\}$ denotes the impulse response of the transfer function $G$ (i.e., $G(z)=$ $\left.\sum_{i=0}^{\infty} \phi_{i} z^{-i}\right)$.

One drawback of using this definition of the $\ell_{1}$ norm is that the $\infty$ norm of a signal only retains the worst-case deviation of the signal, without taking into account if the worst-case negative deviation is different from the worst-case positive deviation. This increases the conservativeness of the controllers designed based on minimizing the $\ell_{1}$ norm.

3.2. Asymmetric objective functional. To solve this drawback, the following objective functional (denoted by $\varphi(w)$ ) was proposed by the authors (Naib et al. [11]), to give information on the signal amplitude, retaining information on the asymmetricity, and being valid for multivariable systems:

$$
\varphi(w)=\left[\begin{array}{l}
w^{+} \\
w^{-}
\end{array}\right]
$$

where $w^{+}=\max \left\{0, \max _{k}(w(k))\right\}$ and $w^{-}=\max \left\{0,-\min _{k}(w(k))\right\}$. 
By extension for a vector of signals $z \in \mathbb{R}^{n}$, we denote

$$
\varphi(z)=\left[\begin{array}{l}
z^{+} \\
z^{-}
\end{array}\right], \quad \text { where for } i=1, \ldots, n \quad\left(z^{+}\right)_{i}=\left(z_{i}\right)^{+},\left(z^{-}\right)_{i}=\left(z_{i}\right)^{-} .
$$

Hence, $\varphi(z) \leq Z$, where $Z=\left[\begin{array}{c}z_{\max } \\ z_{\min }\end{array}\right]$ is equivalent to $-z_{\min } \leq z \leq z_{\max }$, the inequalities considered componentwise. In the proposed context, this objective functional is used to handle the asymmetrical aspect of any set of bounded signals, as it is now presented.

3.3. Asymmetric analysis. The following result, which is an extension to MIMO systems of the theorem proposed in Naib et al. [11], gives necessary and sufficient conditions for a vector of signals to avoid the constraints, that is, $\varphi(z) \leq Z$ for any bounded vector of inputs $\varphi(w) \leq W$ such that $z=M w$.

THeOREM 3.1. The output vector $z \in \mathbb{R}^{n}$ of an LTI system is constrained in the nonsymmetrical domain defined by $\varphi(z) \leq Z$ for any input vector $w \in \mathbb{R}^{m}$ such that $\varphi(w) \leq W$ if and only if

$$
\Psi W \leq Z,
$$

where

$$
\Psi=\left[\begin{array}{cc}
\sum_{l=0}^{\infty} \phi_{l}^{+} & \sum_{l=0}^{\infty} \phi_{l}^{-} \\
\sum_{l=0}^{\infty} \phi_{l}^{-} & \sum_{l=0}^{\infty} \phi_{l}^{+}
\end{array}\right] ; \quad\left[\phi_{l}^{-}\right]_{i, j}=\max \left\{-\left[\phi_{l}\right]_{i, j}, 0\right\},
$$

and $\left\{\phi_{l} \in \mathbb{R}^{n \times m}, l=0, \ldots, \infty\right\}$ denote the elements of the impulse response of the matrix transfer function $M$ of the system (i.e., $z=M w$ ).

Remark 3.2. If the constraints were symmetric $\left(w_{\min }=w_{\max }\right.$ and $\left.z_{\min }=z_{\max }\right)$, then the second set of rows in the definition of $\Psi$ is redundant, and the standard $\ell_{1}$ condition problem is obtained: $\sum_{l=0}^{\infty}\left(\phi_{l}^{+}+\phi_{l}^{-}\right) w_{\max } \leq z_{\max }$.

3.4. Controller design for asymmetric signals. The following controller design technique is now discussed: calculate a stabilizing controller $K$ that minimizes a certain generalized output vector $z_{1}$ when the generalized input signal $w$ is bounded in magnitude and hard limitations are imposed on an output vector $z_{2}$ (in the hydrogen reformer $w$ corresponds to a vector composed by the reference and the disturbance signals, $z_{2}$ is the control signal, and $z_{1}$ corresponds to the measured output deviations). This problem can be expressed mathematically as follows:

$$
\min _{\varphi(w) \leq W}\left\|\varphi\left(z_{1}\right)\right\|_{\infty} \quad \text { s.t. } \varphi\left(z_{2}\right) \leq Z_{2} .
$$

We denote by $M(K)$ the matrix transfer function from the generalized input vector $w$ to the generalized output vector $z_{1}$. This $M(K)$ is usually composed of several characteristic (matrix) transfer functions, such as the sensitivity $S=(1+K G)^{-1}$, the control 
sensitivity $M=K S$, the complementary sensitivity $T=I-S$, and so forth, and maybe weighted by transfer functions $W_{i}$ given by the design specifications, as is usual in robust control. Then, using the result in the previous section, (3.6) can be expressed as calculating a stabilizing controller $K$ that solves the following minimization problem:

$$
\min _{K}\left\|\Psi_{1}(K) W\right\|_{\infty} \quad \text { s.t. } \Psi_{2} W \leq Z \leq Z_{2}
$$

The good news is that (3.7) can be transformed to a LP problem, following a method parallel to that already proposed for solving the following $\ell_{1}$ optimization:

$$
\min _{K}\left\|M_{1}(K)\right\|_{1} \quad \text { s.t. }\left\|M_{2}(K)\right\|_{1} \leq Z_{2} \text {. }
$$

where $M_{1}$ and $M_{2}$ are the matrix transfer functions from $w$ to $z_{1}$ and $z_{2}$, respectively.

Unfortunately the description of the resulting LP problem in the general case is too cumbersome: as it corresponds to small modifications on the $\ell_{1}$ optimization technique only a short sketch is now presented for a one-block SISO problem, where only the main modifications are discussed. The detailed example in next section should clarify any remaining issue.

The original $\ell_{1}$ optimization problem (3.8) can be solved by separating the positive and negative terms of the corresponding matrix of impulse responses (Dahleh and Pearson [5]):

$$
M_{1}=\sum_{i=0}^{\infty} \phi_{1 i}^{+} z^{-i}-\sum_{i=0}^{\infty} \phi_{1 i}^{-} z^{-i}, \quad M_{2}=\sum_{i=0}^{\infty} \phi_{2 i}^{+} z^{-i}-\sum_{i=0}^{\infty} \phi_{2 i}^{-} z^{-i} .
$$

The following LP problem is then obtained:

$$
\min _{\varepsilon_{j}, \Phi_{1 i}^{+}, \Phi_{1 i}^{-}, \Phi_{2 i}^{+}, \Phi_{2 i}^{-}} \sum_{j=0}^{m} \varepsilon_{j} \quad \text { s.t. } \sum_{i=0}^{\infty} \phi_{1 i}^{+}+\sum_{i=0}^{\infty} \phi_{1 i}^{-} \leq \varepsilon, \sum_{i=0}^{\infty} \phi_{2 i}^{+}+\sum_{i=0}^{\infty} \phi_{2 i}^{-} \leq Z_{2 \max } .
$$

The first set of constraints in (3.10) are the so-called norm constraints; the second set correspond to the signal constraints, with additional interpolation, feasibility, and rank constraints that are equivalent for both problems, so they are omitted for clarity.

In the presence of asymmetric signal, the proposed functional can be used, being only necessary to replace the norm constraint in (3.10) given by the $\ell_{1}$ norm with the constraint given the objective functional presented in the previous section, obtaining the following LP:

$$
\min _{\varepsilon_{j}^{+}, \varepsilon_{j}^{-}, \Phi_{1 i}^{+}, \Phi_{1 i}^{-}, \Phi_{2 i}^{+}, \Phi_{2 i}^{-}}\left(\sum_{j=0}^{m} \varepsilon_{j}^{+}+\sum_{j=0}^{m} \varepsilon_{j}^{-}\right) \quad \text { s.t. } \Psi_{1} W \leq \varepsilon, \Psi_{2} W \leq Z_{2},
$$

where $\varepsilon=\left[\varepsilon^{+}, \varepsilon^{-}\right]^{t}$, and $\varepsilon^{+}, \varepsilon^{-} \in \mathbb{R}^{m}$ are column vectors with dimensions equal to the number of generalized outputs in $z_{1}: \mathcal{\varepsilon}^{+}=\left\{\varepsilon_{j}^{+}\right\}_{j=0}^{m}, \mathcal{\varepsilon}^{-}=\left\{\varepsilon_{j}^{-}\right\}_{j=0}^{m}$. 
The example in the next section clarifies the proposed method.

\section{Regulator design for a hydrogen reformer}

This section studies the application of the controller design technique proposed in Section 3 to the hydrogen reformer control problem presented in Section 2. The design will be based on solving a mixed sensitivity problem in the proposed objective functional, with an additional constraint given by the control signal saturations. The transformation into a linear programming problem will be presented in detail. The solution of this LP will give the desired controller.

4.1. Control specifications. The main objectives to attend are the following.

(i) To reduce the effect of the unknown but nonsymmetrical bounded disturbances in the output signal.

(ii) To weight the effect of the reference signal in the output signal.

(iii) To respect the constraints on the control signal. In our problem there are asymmetric bounds in the control signal $u$ as $-u_{\min } \leq u(k) \leq u_{\max }$. Using the objective functional proposed, this can be written as $\varphi(u) \leq U$, where $U^{t}=\left[u_{\max }, u_{\min }\right]$. Thus, the control signal is constrained to evolve in an asymmetrical set $\Omega$, given by

$$
u \in \Omega=\left\{u(k) \in \mathbb{R} / \varphi(u) \leq U ; U=[0.6,2.27]^{t}\right\}
$$

(iv) Moreover, the expected amplitude of the disturbance signal is known to be asymmetric (in our example, decreases in temperature are bigger than positive deviations). This means that $d(k)$ is such that $-d_{\min } \leq d(k) \leq d_{\max }$ which can be rewritten as $\varphi(d) \leq D$, where $D^{t}=\left[d_{\max }, d_{\min }\right]$. Then, the disturbance signal affecting this plant belongs to the following asymmetrical set:

$$
d \in \mathscr{D}=\left\{d(k) \in \mathbb{R} / \varphi(d) \leq D ; D=[1.9,0.1]^{t}\right\} .
$$

(v) In addition to this, the expected variations in the reference signal are known a priori: the reference is asymmetrically bounded by $-R_{\min } \leq r(k) \leq R_{\max }$, or, in more compact form using the proposed objective functional, this can be rewritten as $\varphi(r) \leq R$. Thus, the reference signal can be assumed to be constrained in the following set:

$$
r \in \mathfrak{R}=\left\{r(k) \in \mathbb{R} / \varphi(r) \leq R ; R=[1,5]^{t}\right\} .
$$

In summary, all of these problems can be stated as designing a controller that ensures that $\varphi(u) \leq U$ when $\varphi(d) \leq D$ and $\varphi(r) \leq R$, while minimizing the output deviation.

4.2. Transformation into a linear programming problem. The transformation into an LP is now discussed for the general problem. 
Following the usual method in robust control, the control objectives can be achieved by solving (3.6), where $z_{2} \equiv u, z_{1} \equiv y$, and $w \equiv\left[W_{1} r W_{2} d\right]^{t}$. This can be expressed as the following mixed sensitivity problem.

Search among all the internally stabilizing controllers for the one which minimize the following cost function:

$$
\min \left(\varphi\left[\begin{array}{ll}
W_{1} S & W_{2} T
\end{array}\right]\right) \quad \text { s.t. } \varphi(K S)(R+\bar{D}) \leq U
$$

where $S=1 /(1+K G)$ represents the sensitivity, $K$ is the controller, $T=K G /(1+K G)$ is the complementary sensitivity, and $W_{1}$ and $W_{2}$ are weighting transfer functions.

Since the control signal is given by $u=K S(r-d)$, then the constraints are respected if the following inequality is fulfilled:

$$
\varphi(K S)(R+\bar{D}) \leq U
$$

where $\bar{D}=[0.1,1.9]^{t}$ represents the constraint vector on the signal $-d$.

To simplify the transformation into an LP, in this study the optimization problem is expressed directly on the coefficients of the impulse response of the considered transfer functions (Dahleh and Pearson [5], Tadeo and Grimble [14]): if we denote the impulse responses of $W_{1} S, W_{2} T$, and $K S$, respectively, to be $\phi_{1}, \phi_{2}$, and $\phi_{3}$, then, using the previous formalism, our problem can be rewritten as

$$
\begin{aligned}
& \min _{\varepsilon_{1}^{+}, \varepsilon_{1}^{-}, \varepsilon_{2}^{+}, \varepsilon_{2}^{-}, \Phi_{1 i}^{+}, \Phi_{1 i}^{-}, \Phi_{2 i}^{+}, \Phi_{2 i}^{-}, \Phi_{3 i}^{+}, \Phi_{3 i}^{-}}\left(\varepsilon_{1}^{+}+\varepsilon_{1}^{-}+\varepsilon_{2}^{+}+\varepsilon_{2}^{-}\right) \\
& \text {s.t. }\left\{\begin{array}{l}
\sum_{i=0}^{\infty}\left(\phi_{1 i}^{+} d_{\max }+\phi_{1 i}^{-} d_{\min }\right) \leq \varepsilon_{1}^{+}, \\
\sum_{i=0}^{\infty}\left(\phi_{1 i}^{-} d_{\min }+\phi_{1 i}^{+} d_{\max }\right) \leq \varepsilon_{1}^{-}, \\
\sum_{i=0}^{\infty}\left(\phi_{2 i}^{+} r_{\max }+\phi_{2 i}^{-} r_{\min }\right) \leq \varepsilon_{2}^{+}, \\
\sum_{i=0}^{\infty}\left(\phi_{2 i}^{+} r_{\min }+\phi_{2 i}^{-} r_{\max }\right) \leq \varepsilon_{2}^{-}, \\
\sum_{i=0}^{\infty}\left(\phi_{3 i}^{+}\left(r_{\max }+d_{\min }\right)+\phi_{3 i}^{-}\left(r_{\min }+d_{\max }\right)\right) \leq u_{\max }, \\
\sum_{i=0}^{\infty}\left(\phi_{3 i}^{+}\left(r_{\min }+d_{\max }\right)+\phi_{3 i}^{-}\left(r_{\max }+d_{\min }\right)\right) \leq u_{\min } .
\end{array}\right.
\end{aligned}
$$

To solve this problem, it is necessary to add some feasibility constraint equalities to make the link between $\phi_{1}, \phi_{2}$, and $\phi_{3}$ :

$$
S+T=1, \quad S+G K S=1 .
$$

These equalities can be rewritten as

$$
\begin{gathered}
d w_{1} * n w_{2} * \phi_{1}+n w_{1} * d w_{2} * \phi_{2}=n w_{1} * n w_{2}, \\
d w_{1} * d g * \phi_{1}+n g * n w_{1} * \phi_{2}=n w_{1} * d g,
\end{gathered}
$$


where $W_{1}=n w_{1} / d w_{1}, W_{2}=n w_{2} / d w_{2}$, and $G=n g / d g$, with $n w_{1}, d w_{1}, n w_{2}, d w_{2}, n g$, and $d g$ coprime polynomials.

Further, additional constraints (or namely interpolation constraints) must be added to ensure the internal stability of the system, as in standard $\ell_{1}$ optimization. These constraints mean that any unstable pole (resp., zero) of the plant cannot be cancelled by any zero (resp., pole) of the controller. If we denote by $\left\{q_{k}, k=1, \ldots, n\right\}$ the unstable zeros of the plant and by $\left\{p_{l}, l=1, \ldots, m\right\}$ its unstable poles, then

$$
\begin{gathered}
K\left(z^{-1}\right) G\left(z^{-1}\right) \|_{z=q_{k}} \text { must be equal to } 0, \\
K\left(z^{-1}\right) G\left(z^{-1}\right) \|_{z=p_{l}} \text { should tend to infinity. }
\end{gathered}
$$

In this problem, taking into account the feasibility constraints, the interpolation constraints are redundant.

The obtained linear programming problem has infinite number of variables and constraints. In order to obtain a finite-dimensional problem, this semi-infinite linear programming problem is truncated as is frequently done in $\ell_{1}$ optimization. The dimension of the new linear programming problem, denoted by $\mu$, can be calculated from the dual problem. Finally, we have a linear programming problem in $3 \mu+4$ variables which can be summarized as follows:

$$
\min c^{t} x \quad \text { s.t. } A x \leq b, A_{e q} x=b_{e q} \text {, }
$$

where $x$ is formed by the variables $\varepsilon_{1}^{+}, \varepsilon_{2}^{+}, \varepsilon_{1}^{-}, \varepsilon_{2}^{-}, \phi_{1}^{+}, \phi_{1}^{-}, \phi_{2}^{+}, \phi_{2}^{-}, \phi_{3}^{+}$, and $\phi_{3}^{-}$. The vector $c$ is such that $c^{t} x=\varepsilon_{1}^{+}+\varepsilon_{2}^{+}+\varepsilon_{1}^{-}+\varepsilon_{2}^{-}$. Matrices $A, A_{e q}$ and vectors $b$ and $b_{e q}$ are given according to the linear programming problem defined in (4.6).

4.3. Controller calculation. The linear model of the plant that will be used to design the controller is

$$
\begin{array}{r}
G\left(z^{-1}\right)=\frac{-0.032 z^{-1}\left(1+0.2453 z^{-1}\right)\left(1-0.623257 z^{-1}\right)\left(1+0.9999 z^{-1}\right)}{\left(1+0.58958 z^{-1}\right)\left(1-0.615995 z^{-1}\right)\left(1+0.81983 z^{-1}\right)} \\
\frac{\left(1+15.4484 z^{-1}\right)\left[\left(1+0.788216 z^{-1}\right)^{2}+1.566429^{2}\right]}{\left(1-0.910085 z^{-1}\right)\left[\left(1+0.419267 z^{-1}\right)^{2}+0.379915^{2}\right]} .
\end{array}
$$

The model was obtained in transfer-function form from the input ( $u$ : fuel-flow setpoint) to the output ( $y$ : outlet temperature) and includes the flow controller dynamics. In this case, a simplified model was previously obtained by modeling and identification using data from the real plant (Shakoor, [12]).

Note that this plant has a stable nonminimum phase model with three zeros outside the unit circle, at $z=15.45$ and $z=0.79 \pm 1.57 j$. 
10 Control with asymmetric bounds of a hydrogen reformer

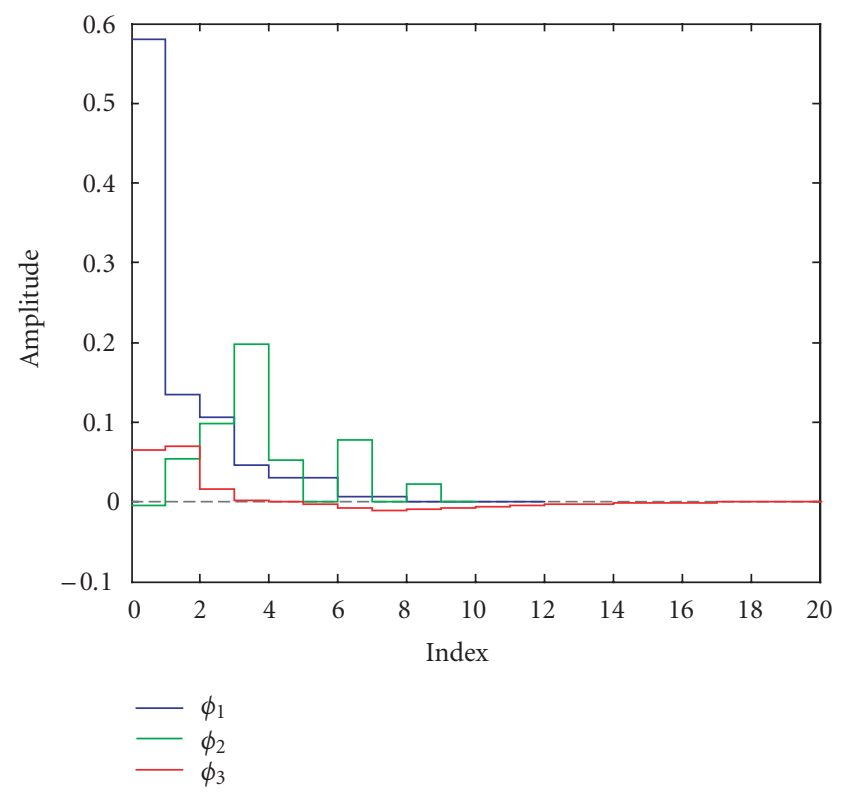

Figure 4.1. The $\Phi_{i}$.

The disturbance model $W_{d}$ was also identified using the available data from the real plant and it is given by

$$
\begin{aligned}
W_{d}\left(z^{-1}\right)=\frac{-0.032 z^{-1}\left(1+0.2453 z^{-1}\right)\left(1-0.623257 z^{-1}\right)}{\left(1+0.58958 z^{-1}\right)\left(1-0.615995 z^{-1}\right)} \\
\frac{\left(1+0.9999 z^{-1}\right)\left(1+15.4484 z^{-1}\right)\left[\left(1+0.788216 z^{-1}\right)^{2}+1.566429^{2}\right]}{\left(1+0.81983 z^{-1}\right)\left(1-0.910085 z^{-1}\right)\left[\left(1+0.419267 z^{-1}\right)^{2}+0.379915^{2}\right]} .
\end{aligned}
$$

The weights $W_{1}$ and $W_{2}$ were selected based on a tradeoff between the specifications obtained from the system analysis and the control effort. These weights are, respectively, the discrete-time equivalents of the following continuous-time transfer functions: $(0.5 s+$ $0.005) /\left(s+10^{-6}\right)$ and $(50 s+0.5) /(0.1 s+1)$ for a sampling time $T_{s}=30$.

This linear problem can be solved with the "linprog" optimization function of Matlab, for increasing values of $\mu$ until no improvement is obtained. The obtained impulse responses for $\mu=20$ are shown in Figure 4.1.

The characteristic transfer functions $S, T$, and $M$ can be computed from any $\phi_{i}$. The frequency responses are shown in Figure 4.2.

The controller can be calculated from the relation that defines any of the characteristic transfer functions. For example, using the relation which defines the sensitivity $\left(S=(1+K G)^{-1}\right)$, one can compute the corresponding regulator from $K=G^{-1}\left(S^{-1}-1\right)$, 
M. Naib et al. 11
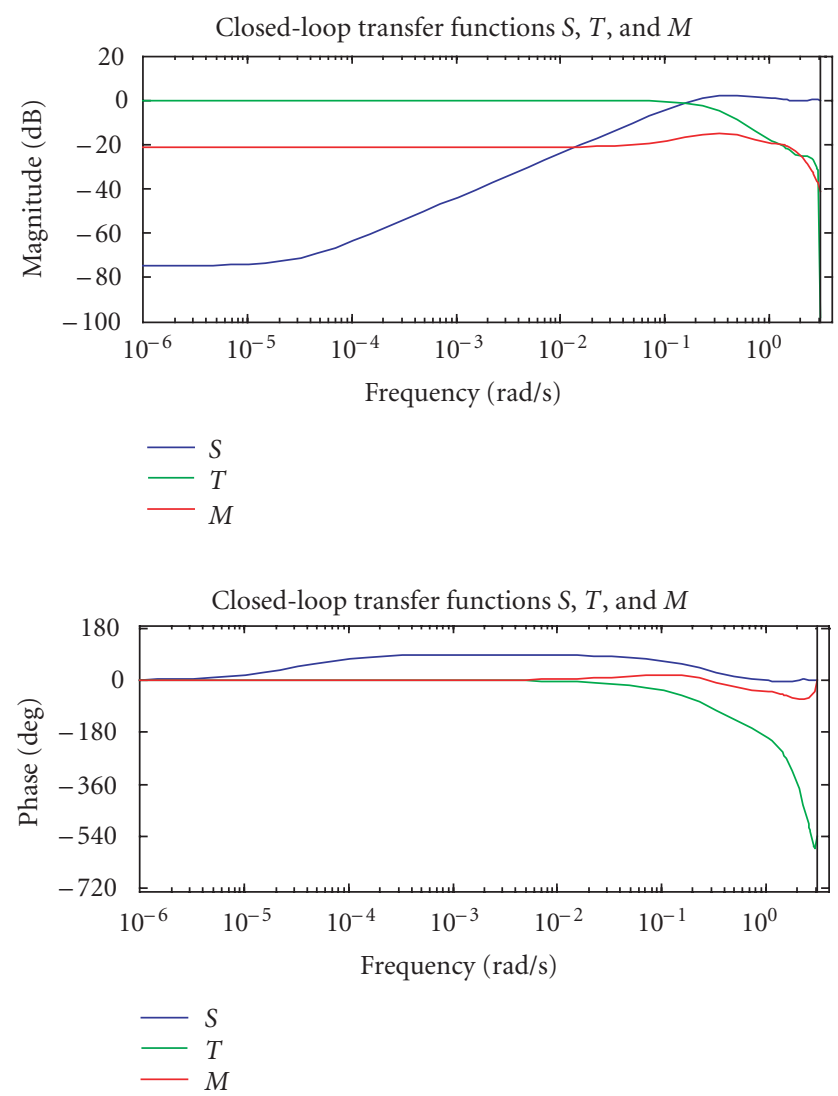

Figure 4.2. Frequency response of the closed-loop transfer functions $S, T$, and $M$.

giving

$$
\begin{aligned}
K\left(z^{-1}\right)=\frac{0.0642\left(1-0.9101 z^{-1}\right)\left(1+0.9898 z^{-1}\right)\left(1+0.8198 z^{-1}\right)}{\left(1-z^{-1}\right)\left(1+0.9999 z^{-1}\right)\left(1-0.6233 z^{-1}\right)} \\
\\
\frac{\left(1-0.6160 z^{-1}\right)\left(1+0.5896 z^{-1}\right)\left[\left(1-0.4485 z^{-1}\right) 2+0.4968^{2} z^{-2}\right]}{\left(1+0.5593 z^{-1}\right)\left(1+0.2453 z^{-1}\right)\left[\left(1-0.4235 z^{-1}\right) 2+0.38022 z^{-2}\right]} \\
\\
\frac{\left[\left(1+0.0716 z^{-1}\right) 2+0.5079^{2} z^{-2}\right]\left[\left(1+0.4193 z^{-1}\right) 2+0.3799^{2} z^{-2}\right]}{\left[\left(1-0.0204 z^{-1}\right) 2+0.47382 z^{-2}\right]\left[\left(1+0.2806 z^{-1}\right) 2+0.44802 z^{-2}\right]} .
\end{aligned}
$$

Since the controller is of high order (11th order), to make it feasible for implementation, it is necessary to calculate a reduced-order controller which maintains the same performances of the original controller. For this, we can use, for example, the "invfreqz" function of Matlab. In Figure 4.3, the frequency response of the reduced-order regulator 
12 Control with asymmetric bounds of a hydrogen reformer
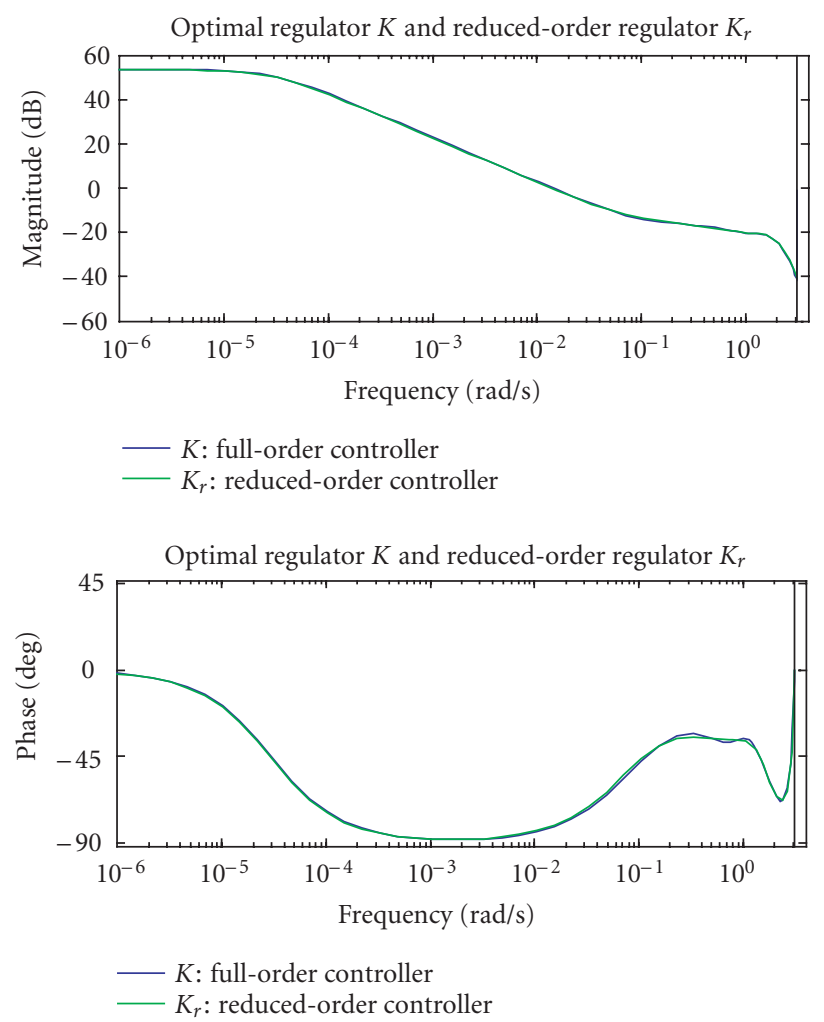

Figure 4.3. Frequency response of the optimal controller $K$ and the reduced-order controller $K_{r}$.

is depicted on the original controller. It is possible to see that over the 4th order the two controllers give a similar response, so a 4 th-order controller is selected.

\section{Conclusion}

In this paper, a new way to handle asymmetrical signals has been presented. Using a new asymmetric objective functional, instead of the usual peak-to-peak norm, necessary and sufficient conditions are given to check if some performance specifications are fulfilled. In the next step, the analysis and the synthesis problems are also formulated with respect to this new objective functional. As in the $\ell_{1}$-optimal control, this approach leads to a linear programming problem which can be solved by available optimization tools. Compared with the linear problem obtained using standard $\ell_{1}$-optimal control, no additional variables are added to the primal problem, but the number of constraints is doubled, to cope with the asymmetricity. The technique has been demonstrated on a system from petrochemical industry: a hydrogen reformer.

\section{Acknowledgment}

This work has been funded by Morocco-Spain Research Project AECI 23P/00, 8/03/P and CICYT Project DPI2004-07444-C04-02. 


\section{References}

[1] A. Benzaouia and C. Burgat, Regulator problem for linear discrete-time systems with nonsymmetrical constrained control, International Journal of Control 48 (1988), no. 6, 2441-2451.

[2] F. Blanchini and M. Sznaier, Persistent disturbance rejection via static-state feedback, IEEE Transactions on Automatic Control 40 (1995), no. 6, 1127-1131.

[3] M. A. Dahleh and I. J. Diaz-Bobillo, Control of Uncertain Systems: A Linear Programming Approach, Prentice-Hall, New Jersey, 1995.

[4] M. A. Dahleh and J. B. Pearson Jr., $\ell_{1}$-optimal feedback controllers for MIMO discrete-time systems, IEEE Transactions on Automatic Control 32 (1987), no. 4, 314-322.

[5] __ Optimal rejection of persistent disturbances, robust stability, and mixed sensitivity minimization, IEEE Transactions on Automatic Control 33 (1988), no. 8, 722-731.

[6] M. Dambrine, J. P. Richard, and P. Borne, Feedback control of time-delay systems with bounded control and state, Mathematical Problems in Engineering 1 (1995), no. 1, 77-87.

[7] Y. Eun, C. Gökçek, P. T. Kabamba, and S. M. Meerkov, An LQG approach to systems with saturating actuators and anti-windup implementation, Mathematical Problems in Engineering 8 (2002), no. $4-5,311-321$.

[8] P. O. Malaterre and M. Khammash, $\ell_{1}$ controller design for a high-order 5-pool irrigation canal system, Proceedings of the Conference on Decision and Control, Sydney, 2000.

[9] F. Mesquine, F. Tadeo, and A. Benzaouia, Regulator problem for linear systems with constraints on control and its increment, Proceedings of the 15th IFAC World Congress, Barcelona, 2001.

[10] _ Regulator problem for linear systems with constraints on control and its increment or rate, Automatica 40 (2004), no. 8, 1387-1395.

[11] M. Naib, A. Benzaouia, and F. Tadeo, $\ell_{1}$-control using linear programming for systems with asymmetric bounds, International Journal of Control 78 (2005), no. 18, 1459-1465.

[12] A. S. Shakoor, Industrial uses of predictive control, MSc. dissertation, University of Strathclyde, Glasgow, 1996.

[13] V. F. Sokolov, Adaptive $\ell_{1}$ robust control for SISO systems, Systems \& Control Letters 42 (2001), no. 5, 379-393.

[14] F. Tadeo and M. J. Grimble, Advanced control of a hydrogen reformer, IEE Computing and Control Engineering Journal 13 (2002), 305-314.

[15] _ Controller design using linear programming for systems with constraints. Part 1: tutorial introduction; part 2: controller design; part 3: design examples, IEE Computing and Control Engineering Journal, 12 (2002), 273-276, 13 (2003), 49-52, 89-93.

[16] F. Tadeo, A. Holohan, and P. Vega, $\ell_{1}$-optimal regulation of a $\mathrm{pH}$ control plant, Computers \& Chemical Engineering 22 (1998), suppl. 1, S459-S466.

[17] M. Vidyasagar, Optimal rejection of persistent bounded disturbances, IEEE Transactions on Automatic Control 31 (1986), no. 6, 527-534.

M. Naib: Department of Physics, Faculty of Sciences Semlalia, Cadi Ayyad University BP 2390,

Marrakech 40000, Morocco

E-mail address: m.naib@ucam.ac.ma

F. Tadeo: Departamento Ingeniería de Sistemas y Automática, Universidad de Valladolid, 47005 Valladolid, Spain

E-mail address: fernando@autom.uva.es

A. Benzaouia: Department of Physics, Faculty of Sciences Semlalia, Cadi Ayyad University BP 2390,

Marrakech 40000, Morocco

E-mail address: benzaouia@ucam.ac.ma 


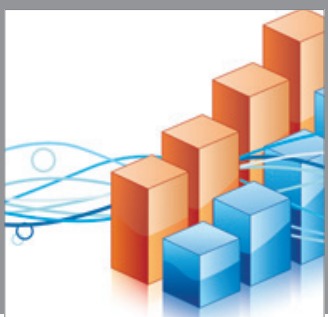

Advances in

Operations Research

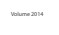

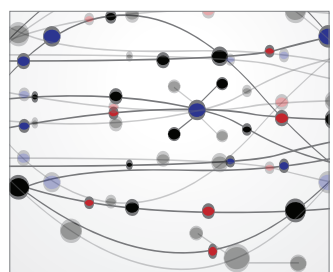

\section{The Scientific} World Journal
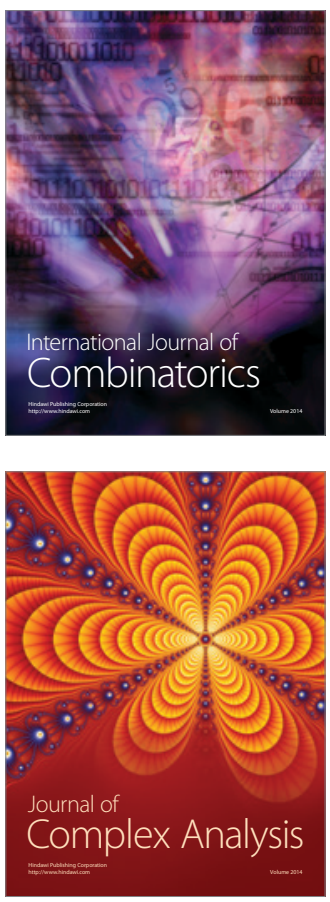

International Journal of

Mathematics and

Mathematical

Sciences
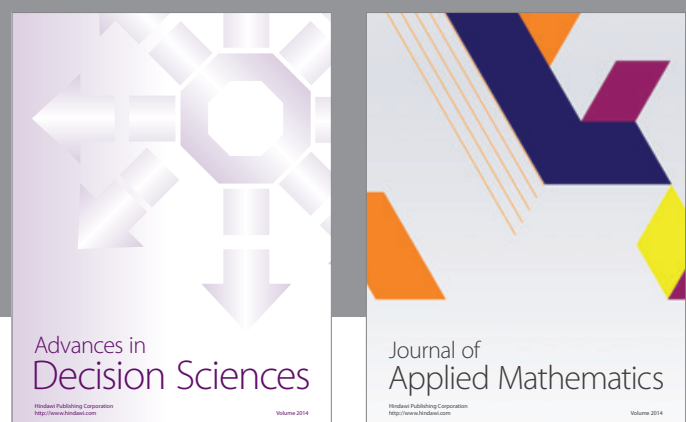

Journal of

Applied Mathematics
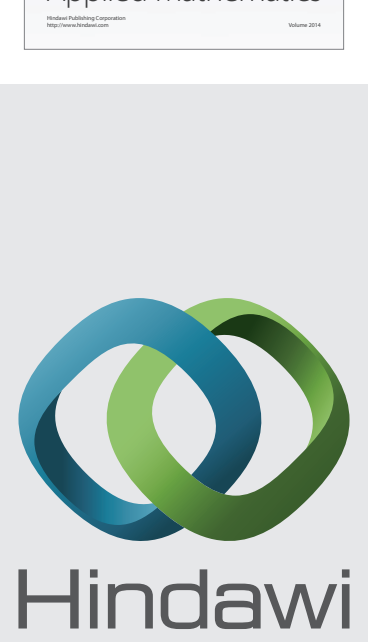

Submit your manuscripts at http://www.hindawi.com
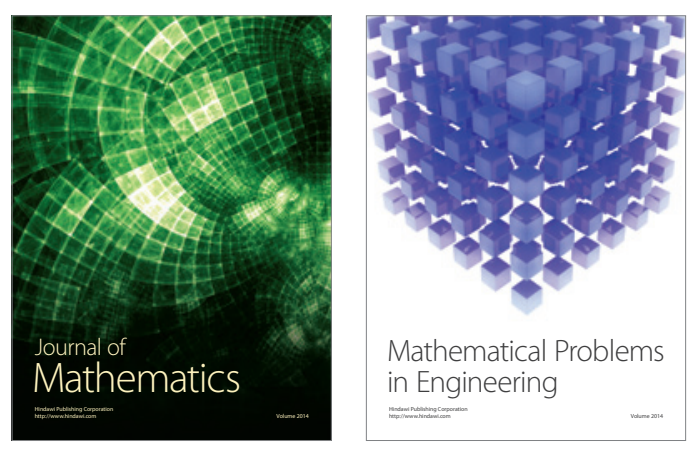

Mathematical Problems in Engineering
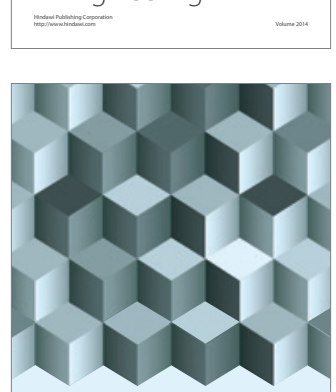

Journal of

Function Spaces
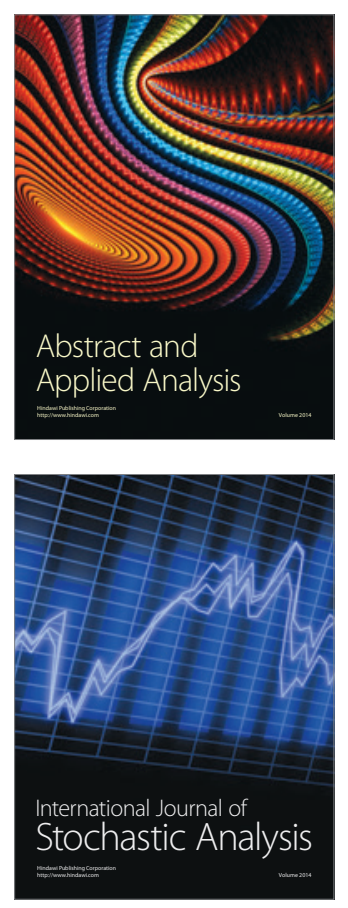

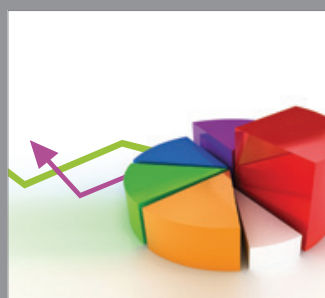

ournal of

Probability and Statistics

Promensencen
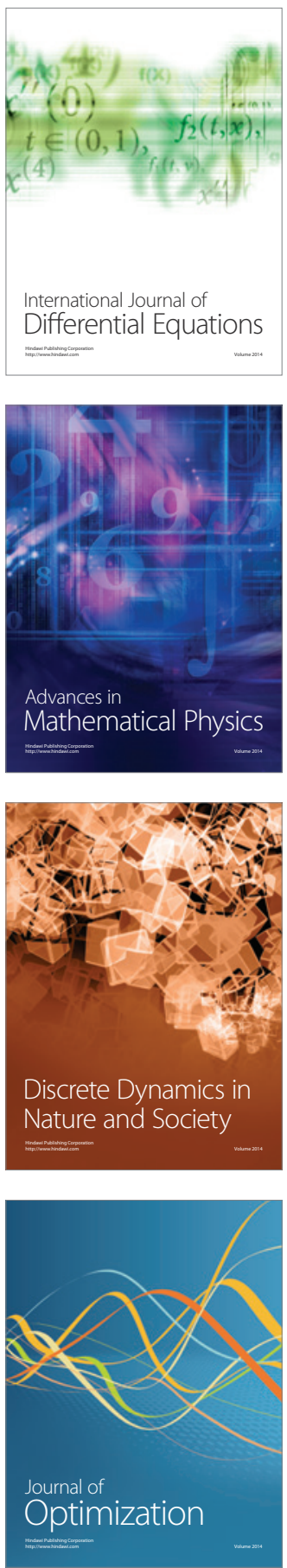